\title{
Retranscrire la violence et le traumatisme
}

DOI:

10.3917/ving. 139.0165

\section{Document Version}

Accepted author manuscript

Link to publication record in Manchester Research Explorer

\section{Citation for published version (APA):}

Burgard, A. (2018). Retranscrire la violence et le traumatisme: Mises en récit administratives de la persécution dans l'immédiate après-Shoah. Vingtieme Siecle: Revue d'Histoire, 139(3), 165.

https://doi.org/10.3917/ving.139.0165

\section{Published in:}

Vingtieme Siecle: Revue d'Histoire

\section{Citing this paper}

Please note that where the full-text provided on Manchester Research Explorer is the Author Accepted Manuscript or Proof version this may differ from the final Published version. If citing, it is advised that you check and use the publisher's definitive version.

\section{General rights}

Copyright and moral rights for the publications made accessible in the Research Explorer are retained by the authors and/or other copyright owners and it is a condition of accessing publications that users recognise and abide by the legal requirements associated with these rights.

\section{Takedown policy}

If you believe that this document breaches copyright please refer to the University of Manchester's Takedown Procedures [http://man.ac.uk/04Y6Bo] or contact uml.scholarlycommunications@manchester.ac.uk providing relevant details, so we can investigate your claim.

\section{open 2 Access}




\title{
Retranscrire la violence et le traumatisme
}

\author{
Mises en récit administratives de la persécution dans l'immédiate \\ après-Shoah
}

\author{
Antoine Burgard
}

\begin{abstract}
À la fin des années 1940, plus de 1000 orphelins de la Shoah ont pu partir au Canada dans le cadre d'un projet du Canadian Jewish Congress. Leurs dossiers de demande de visa révèlent comment les travailleuses sociales et humanitaires, qui remplissent ces demandes en Europe, retranscrivent la violence et le traumatisme de la guerre et formulent des solutions pour le «retour à la normale» des jeunes rescapés. Lieu d'observation des discours et des pratiques des adultes, cette archive recèle aussi des « éclats de réel » qui permettent de contribuer à une histoire de l'expérience enfantine et adolescente de la persécution.
\end{abstract}

L'enthousiasme suscité par la numérisation et la mise à disposition dans plusieurs pays des archives de l'International Tracing Service (ITS) témoigne de l'intérêt croissant que portent les historiens et historiennes de la Shoah au traitement sériel de sources nominatives ${ }^{1}$. Si les fonds de l'ITS sont d'une ampleur sans pareille, d'autres archives possèdent des corpus relativement proches. C'est notamment le cas des Archives juives canadiennes Alex-Dworkin (CJA) à Montréal qui ont conservé plus de mille dossiers individuels (case files ${ }^{2}$ ) d'orphelins de la Shoah ayant immigré au Canada entre septembre 1947 et mars 1952. Ces orphelins sont partis dans le cadre du War Orphans Project, un programme d'accueil mis en place par le Canadian Jewish Congress (CJC), alors principale association juive du pays ${ }^{3}$. Venus principalement d'Europe centrale et orientale, beaucoup ont connu la déportation en Allemagne, en Autriche et en Pologne, ont passé la guerre cachés dans les campagnes belges, françaises ou polonaises ou ont fui en Union soviétique ou en Angleterre. À la sortie de la guerre, leurs parcours sont tout aussi hétérogènes. Certains vivent dans les camps de personnes déplacées d'Allemagne et d'Autriche, d'autres retournent dans leur ville natale. D'autres encore partent en Angleterre, en Belgique, en France ou en Suède ou tentent de rejoindre la Palestine ou les États-Unis. En écrivant l'histoire de cette cohorte à travers les

\footnotetext{
${ }^{1}$ Sur l'ITS, voir la contribution de Diane Afoumado dans ce numéro; voir également Suzanne BrownFleming, Nazi Persecution and Postwar Repercussions: The International Tracing Service Archive and Holocaust Research, Lanham, Rowman \& Littlefield, 2016.

${ }^{2}$ Les historiennes canadiennes Franca Iacovetta et Wendy Mitchinson proposent une définition large du case file comme «les documents produits par des institutions politiques, sociales, légales et autre, chargées de catégoriser et d'évaluer certaines populations, souvent avec l'objectif de superviser, soigner, punir et/ou redresser des individus ou des groupes considérés comme étant d'une manière ou d'une autre déviants ou victimes »; Franca Iacovetta et Wendy Mitchinson, «Introduction », in Franca Iacovetta et Wendy Mitchinson (dir.), On the Case : Explorations in Social History, Toronto, University of Toronto Press, 1998, p. 3-21, p. 3 ; sur les usages du dossier en histoire, voir aussi Claire Zalc, Dénaturalisés : les retraits de nationalité sous Vichy, Paris, Éd. du Seuil, 2016 ; Laure Fourtage, «Regards croisés sur le travail social juif : historiens et praticiens face aux dossiers personnels », Hommes \& Migrations, 1312, 2015, p. 130-132.

${ }^{3}$ Pour un survol de ce projet, voir Benjamin Lappin, The Redeemed Children, Toronto, University of Toronto Press, 1963 ; Fraidie Martz, Open your Hearts, Montreal, Vehicule Press, 1996. Voir également Adara Goldberg, Holocaust Survivors in Canada: Exclusion, Inclusion, Transformation, 1947-1955, Winnipeg, University of Manitoba Press, 2015, p. 75-101.
} 
dossiers, il est possible de saisir la diversité des trajectoires de guerre et de sortie de guerre de ces jeunes et de contribuer à une topographie globale des survivants de la Shoah.

Les dossiers doivent aussi être appréhendés comme un lieu d'observation des discours et des pratiques des adultes qui les remplissent puisqu'ils donnent avant tout à voir l'orphelin tel qu'il est compris et construit par les travailleuses sociales et humanitaires qui se font les intermédiaires de l'administration ${ }^{4}$. En établissant l'identité de l'orphelin, en attestant son expérience de la persécution et en planifiant son avenir, ces dernières produisent une écriture administrative singulière de la Shoah où la violence et le traumatisme apparaissent plus ou moins explicitement. Après une brève présentation du corpus, le présent article se propose d'analyser cette mise en récit précoce du génocide. Comment se raconte l'entreprise d'extermination des juifs d'Europe deux ans à peine après la libération des camps et la fin de la guerre ? Quelle est la place accordée au traumatisme et à la souffrance dans les dossiers ? Quelles sont les solutions formulées pour accompagner les jeunes survivants dans ce qui est présenté comme un « retour à la normale » ? Et que révèlent les tensions et les contradictions dans la manière d'écrire la persécution ? Le dossier est donc ici appréhendé comme la mise en récit d'un individu par un tiers, une présentation de l'autre qui répond aux normes fixées par l'administration. Son usage amène néanmoins une autre question : est-il possible de saisir à travers cette archive, si ce n'est le « regard direct ${ }^{5} »$, des «éclats de réel ${ }^{6}$ » qui nous éclaireraient sur les émotions et les modes de pensée de ceux qui en sont l'objet? En d'autres termes, peut-on considérer le dossier et «l'obligation de se raconter ${ }^{7}$ » qui l'accompagne comme un témoignage précoce de l'orphelin, certes fortement encadré et médié mais néanmoins précieux ?

L'ensemble des dossiers des orphelins ayant obtenu un visa dans le cadre du projet ont été conservés aux CJA et ont été dépouillés dans le cadre de ma thèse pour le doctorat d'histoire ${ }^{8}$. Ont été recensés 1115 dossiers acceptés auxquels s'ajoutent 217 dossiers refusés ou abandonnés, également conservés. Plusieurs formulaires existent, mais deux modèles dominent, celui de l'International Refugee Organization (IRO) et celui de l'American Jewish Joint Distribution Committee (AJDC) ${ }^{9}$. Ils comportent une présentation détaillée de l'orphelin, de sa famille et de ses potentiels proches survivants, de son parcours en temps de guerre, une description physique et une photographie, un dossier médical, des renseignements sur sa scolarité et sur son projet professionnel. On y trouve également différentes sections sur l'attitude de l'orphelin par rapport à son possible départ au Canada et des recommandations de l'adulte qui a conduit l'entretien, notamment concernant le placement en famille d'accueil. L’immense majorité des formulaires sont rédigés en anglais. Certains sont en français et, plus

\footnotetext{
${ }^{4}$ Le terme «travailleuses sociales et humanitaires » a été utilisé afin d'englober à la fois le personnel présent dans les camps de personnes déplacées d'Allemagne et d'Autriche et celui œuvrant auprès des communautés juives locales, en France, en Angleterre ou en Tchécoslovaquie par exemple. L'immense majorité de ce personnel étant des femmes, le terme est toujours employé au féminin.

${ }^{5}$ Emmanuel Le Roy Ladurie, Montaillou : village occitan de 1294 à 1324, Paris, Gallimard, 1975, p. 9.

${ }^{6}$ Ludivine Bantigny et Jean-Claude Vimont, « Observer les observateurs : les dossiers personnels et leurs usages en histoire », in Ludivine Bantigny et Jean-Claude Vimont (dir.), Sous l'œeil de l'expert: les dossiers judiciaires de personnalité, Montt-Saint-Aignan, Publications des Universités de Rouen et du Havre, 2010, p. 713 , p. 8.

${ }^{7}$ Didier Fassin, «La supplique : stratégies rhétoriques et constructions identitaires dans les demandes d'aide d'urgence », Annales : histoire, sciences sociales, 55 (5), 2000, p. 955-981, p. 957.

${ }^{8}$ Antoine Burgard, "Une nouvelle vie dans un nouveau pays": trajectoires d'orphelins de la Shoah vers le Canada, 1947-1952 », thèse pour le doctorat en histoire réalisée sous la direction de Yolande Cohen et d'Isabelle von Buletzingslowen, Université Lumière Lyon 2/Université du Québec à Montréal, 2017.

${ }^{9}$ L'IRO récupère le mandat sur les camps de personnes déplacées de l'United Nations Rehabilitation and Relief Administration (UNRRA) en 1947 avant d'être remplacée par le haut-commissariat des Nations unies pour les réfugiés en 1952. L'AJDC est la principale organisation de philanthropie et d'action sociale juive américaine. Créée en 1914, elle est encore active aujourd'hui.
} 
rare encore, en hongrois ou en tchèque et accompagnés d'une traduction. Ils ont été constitués par des travailleuses sociales et humanitaires de l'AJDC, de l'IRO et de plusieurs associations juives européennes en France, en Belgique, en Angleterre, en Tchécoslovaquie ou encore en Suède. Les profils de ces femmes sont encore peu connus. La plupart semblent issues des milieux communautaires où se trouvent les enfants. D'autres, américaines ou anglaises bien souvent, sont arrivées en Europe avec l'AJDC à la fin de la guerre. Cette invisibilité s'explique principalement par l'influence considérable des responsables du CJC qui supervisent la sélection des orphelins et la constitution des dossiers de demande de visa. Ces deux responsables, Ethel Ostry et Lottie Levinson, ont des parcours relativement proches ${ }^{10}$. Toutes les deux sont canadiennes (bien qu'Ostry soit née à Odessa), ont suivi des formations en travail social et ont eu de nombreuses expériences professionnelles dans le milieu communautaire juif canadien. Elles arrivent en Europe au cours de l'année 1945, Ostry rejoignant l'United Nations Relief and Rehabilitation Administration (UNRRA) et Levinson l'AJDC. Les deux femmes encadrent la constitution des dossiers pendant la majeure partie du projet et s'efforcent de garantir leur conformité avec les exigences du gouvernement fédéral canadien. Leur influence explique d'ailleurs en grande partie la relative homogénéité du contenu des dossiers. Ce corpus reste néanmoins résolument pluriel et transnational. Son analyse, «au carrefour d'une histoire des pratiques administratives et d'une histoire des trajectoires sociales ${ }^{11} \|$, s'est avérée particulièrement féconde.

Le dossier renseigne d'abord la trajectoire du jeune rescapé : son traitement sériel a permis de contribuer à une topographie globale des circulations migratoires des survivants de la Shoah. Il invite surtout à étudier les pratiques des nombreux protagonistes impliqués dans sa constitution. C'est cette mise en récit de l'orphelin et de sa trajectoire qui nous intéresse ici : elle est totale et a un impact durable. Les formulaires sont en effet lus par les agents d'immigration canadiens qui doivent valider la demande. Ils sont aussi utilisés par les responsables des associations juives au Canada qui s'en servent pour orienter le placement en famille et l'accès à l'emploi des jeunes rescapés. La constitution du dossier de demande de visa nécessite donc non seulement d'établir l'identité de papier du jeune survivant, mais aussi d'attester sa persécution et de cerner sa personnalité. L'analyse du vocabulaire utilisé, notamment dans les sections «histoire de l'enfant depuis la séparation» et « recommandations du travailleur social concernant le placement de l'enfant » des formulaires de l'IRO, et dans les sections « histoire de l'enfant » et « personnalité de l'enfant » de ceux de l'AJDC, fait ressortir comment les adultes perçoivent et retranscrivent l'expérience de la persécution de l'orphelin et son « retour à la normale ». Ici, une attention toute particulière sera portée à la place accordée dans ces sections à la violence de la persécution et au traumatisme.

\section{Écrire la violence et la souffrance en filigrane}

Un des objectifs premiers du dossier est d'attester la persécution du jeune rescapé et de s'assurer de son statut d'orphelin, la perte des deux parents étant un des critères nécessaires pour pouvoir demander un visa canadien. Les formulaires contiennent des sections qui permettent de connaître précisément la chronologie de la guerre de l'individu et les conditions dans lesquelles il est devenu orphelin. Quand le projet canadien débute à l'automne 1947, plus de deux ans se sont écoulés depuis la libération des camps et la fin de la guerre. La typologie utilisée par les travailleuses sociales et humanitaires témoigne pourtant d'une connaissance

\footnotetext{
${ }^{10}$ Voir Sheena Trimble, «Sortir de l'ombre : Canadiennes juives engagées dans le mouvement d'orphelins (1947-1949) », Canadian Jewish Studies/Études juives canadiennes, 24, 2016, p. 124-148.

${ }^{11}$ Anne-Sophie Bruno, Philippe Rygiel, Alexis Spire et Claire Zalc, «Jugés sur pièces : le traitement des dossiers de séjour et de travail des étrangers en France (1917-1984)», Population, 61 (5), 2006, p. 737-762, p. 740.
} 
relativement fine de l'entreprise d'extermination des juifs d'Europe. Le terme « camp de concentration » est celui qui revient le plus régulièrement ${ }^{12}$. Il est utilisé pour Auschwitz, Theresienstadt et Majdanek, mais aussi pour Belzec et Treblinka qui sont souvent indiqués comme lieux probables de la mort d'un ou des deux parents. Si ce terme dissimule parfois la diversité des expériences de la déportation, beaucoup de dossiers font ressortir plus précisément la complexité du réseau de camps nazis. Plusieurs travailleuses différencient ainsi « camp de concentration» et «camp de travail». La plupart du temps, elles se contentent d'ajouter la mention « camp de travail » (labor camp souvent noté «LC» dans les dossiers) entre parenthèses après le nom du complexe ou du camp principal (par exemple « Auschwitz LC »). Le terme «travailleur forcé » (labor worker, slaveworker ou forced laborer) apparaît régulièrement et plusieurs orphelins indiquent même les usines dans lesquelles ils ont été envoyés. Quelques dossiers différencient Birkenau du reste d'Auschwitz, comme celui du jeune Karol L. qui indique avoir été « déporté » à Auschwitz puis « envoyé » à Birkenau avec son frère ${ }^{13}$, mais, bien souvent, les chronologies individuelles n'indiquent que le nom du complexe sans que l'on puisse déterminer dans quel sous-camp s'est retrouvé l'individu. Dans la majorité des cas, Auschwitz est une dénomination vague qui englobe des parcours et des conditions de libération variés : libération dans l'enceinte du camp, libération dans un souscamp du réseau ou bien libération lors d'une marche de la mort. Le degré de détails dans les récits de la persécution varie donc grandement selon les dossiers. Cela peut être le fait des orphelins eux-mêmes, certains étant plus minutieux ou plus bavards lors de l'entretien, ou bien le fait des adultes qui retranscrivent avec plus ou moins de fidélité, résument ou reformulent les récits des jeunes rescapés. Il en est de même pour les nombreuses erreurs et approximations dans les formulaires. À plusieurs reprises, les noms des camps sont mal orthographiés ou mal situés. Pourtant, au-delà des erreurs et des imprécisions, les dossiers brossent déjà, deux ans seulement après la fin de la guerre, un tableau cohérent du génocide des juifs d'Europe et des parcours de ses survivants. Les centres de mise à mort comme Belzec ou Treblinka, les camps de travail, les exécutions sommaires et la dureté de la vie en ghetto sont bien visibles dans les chronologies individuelles et les récits de séparation d'avec les parents.

La violence extrême de la persécution y apparaît cependant de manière singulière, bien souvent dissimulée derrière la concision et le pragmatisme du langage administratif. Les descriptions de la brutalité et des souffrances endurées lors de la séparation d'avec les parents sont laconiques, même pour les orphelins qui ont été témoins directs de leur mort : " tuée sous les yeux de son fils »; «tué en sa présence ». Abraham et Perla B. ont perdu leur mère en Russie, «morte de faim devant eux ${ }^{14}$. Dans la plupart des cas, ne sont que brièvement indiquées la raison et la date du décès : «abattu», "pogrom», " tuée après avoir été dénoncée », « tué après avoir essayé de s'échapper ». Les formulations témoignent souvent de l'incertitude qui entoure le sort des parents, comme Maurice qui pense que son père «a probablement été tué par une bombe en France en $1940^{15}$ ». Même brèves, ces descriptions permettent parfois de saisir la brutalité des exactions, des pogroms et des exécutions sommaires, comme c'est le cas d'Abraham M. dont le père a été assassiné en pleine rue d'une petite ville polonaise en novembre 1941 ou de Jeno S. dont les parents ont été dénoncés par un des locataires de la maison où ils vivaient à Budapest puis ont été abattus près du

\footnotetext{
12 Sur l'histoire de ce terme, voir notamment Dan Stone, Concentration Camps : A Short History, Oxford, Oxford University Press, 2017.

${ }^{13}$ CJA, dossier individuel de Karol et Zoltan L., 28 janvier 1948, boîte 36 ; seuls le prénom et la première lettre du nom de famille de l'orphelin sont indiqués. Les références d'archives comportent la date de signature du dossier et le numéro de la boîte dans laquelle il se trouve. L'ensemble du corpus se trouve aux Archives juives canadiennes (CJA). Les traductions sont les miennes.

${ }^{14}$ CJA, dossiers individuels d'Abraham et Perla B., 15 décembre 1947, boîte 31.

${ }^{15}$ CJA, dossier individuel de Maurice F., 18 novembre 1947, boîte 33.
} 
Danube ${ }^{16}$. Si les chambres à gaz ne sont directement mentionnées qu'à quatre reprises, les fours crématoires reviennent plus régulièrement. Beaucoup d'orphelins disent savoir que l'un ou les deux parents ont été « incinérés [cremated] dans les crematoriums » d'Auschwitz ou de Belzec. Certains sont catégoriques, comme dans le cas de Bela dont la section concernant sa mère indique «Auschwitz, incinérée 04.05.1944 ». D'autres restent plus prudents. Judith A. affirme ainsi que sa mère «a peut-être été incinérée juste après son arrivée au camp ${ }^{17}$ ». Quelques récits sont plus détaillés et contiennent parfois des descriptions explicites. Ida $\mathrm{H}$. raconte ainsi avoir appris que ses parents ont été déshabillés et abattus, probablement lors des pogroms de l'été 1941 perpétrés dans le ghetto de Zloczow, à l'est de la Pologne. « Sa sœur est devenue hystérique, s'est jetée sur ses parents et a été enterrée vivante. Son frère a été abattu le même jour ${ }^{18}$. » Certaines chronologies individuelles permettent également de faire ressortir cette violence extrême. Celle du dossier de David G., jeune Polonais, qui grandit dans le ghetto de Vilna avant d'être déporté à Stutthof en 1943 puis à Buchenwald en 1944, indique ainsi que son père «a été envoyé au crématorium ${ }^{19}$ ". Le garçon s'est ensuite «échappé mais a été repris par six hommes SS qui l'ont torturé. C'était un jour avant la Libération. Il a ensuite appris que sa mère était morte trois jours avant la Libération et sa sœur deux jours avant ». Ces récits plus détaillés sont néanmoins rares puisqu'ils nécessitent que l'orphelin soit prêt à parler et que l'adulte soit prêt à écouter et à retranscrire.

La souffrance est encore plus rarement évoquée dans les récits de la guerre. Par exemple, le dossier de Maria L. indique que la jeune fille a fait face aux «épreuves physiques » et a été « en proie à une grande angoisse ${ }^{20}$ ». Marko R. écrit « avoir énormément souffert de la faim, $\mathrm{du}$ froid et de la terreur ${ }^{21}$ ». Le jeune Tchèque, dont le formulaire est d'ailleurs rempli à la première personne, est un des rares individus à évoquer la faim, qui a pourtant une place centrale pour les survivants, surtout les plus jeunes d'entre eux. Après avoir échappé à une arrestation et s'être enfui de son village, il vit caché avec un cousin pendant plus d'un an et raconte : «Nous avons terriblement souffert de la faim.» La violence et les souffrances subies par les orphelins sont donc directement abordées dans certains dossiers, mais, dans l'ensemble, elles disparaissent derrière la concision du langage des travailleuses sociales et humanitaires. La brutalité de l'expérience concentrationnaire est ainsi souvent implicite, comme dans le dossier de David M., un jeune Tchécoslovaque. La travailleuse humanitaire note uniquement que le garçon a travaillé au «crematorium » d'Auschwitz quand il avait 14 ans ${ }^{22}$. Cette concision est une des constantes du corpus. Peut-être plus que de la pudeur ou du déni, elle rappelle surtout que la finalité du dossier était principalement d'établir le statut d'orphelin et la persécution de l'individu. Le contexte de rédaction et le formulaire en luimême laissant peu de place à l'épanchement, les travailleuses sociales et humanitaires n'évoquent donc dans la plupart des cas la violence et la souffrance que lorsque cela permet d'attester avec certitude la perte des deux parents. Cette écriture administrative ne signifie pas pour autant que le traumatisme est passé sous silence dans les dossiers.

\section{Mettre l'expérience traumatique entre parenthèses}

Dans le dossier de Joseph B., la déportation et la perte de ses parents sont décrites comme une «expérience triste ${ }^{23}$ ». Celui de Meyer W. parle d'un «passé tragique ${ }^{24}$ » et celui de

16 CJA, dossier individuel d'Abraham M., 25 novembre 1947, boîte 37 ; dossier individuel de Jeno S., 17 août 1948 , boîte 39 .

${ }^{17}$ CJA, dossier individuel de Judith A., 16 juin 1948, boîte 31.

${ }^{18}$ CJA, dossier individuel d'Ida H., 2 mars 1948, boîte 35.

${ }^{19}$ CJA, dossier individuel de David G., 8 décembre 1947, boîte 34 .

${ }^{20}$ CJA, dossier individuel de Maria L., 12 avril 1948, boîte 37.

${ }^{21}$ CJA, dossier individuel de Marko R., 23 février 1948, boîte 38.

${ }^{22}$ CJA, dossier individuel de David M., $1^{\text {er }}$ octobre 1947, boîte 37.

${ }^{23}$ CJA, dossier individuel de Joseph B., 2 octobre 1948, boîte 32. 
Martin H. mentionne brièvement la «terrible expérience des camps ${ }^{25}$ ». Maria et Sandor parlent de «[leur] années de souffrance ${ }^{26} »$. Cette expression du traumatisme s'articule souvent autour de l'immense vide laissé par la mort des parents et des proches qui justifie les envies de départ. L'insistance des responsables du CJC et de leurs collaborateurs sur l'importance d'une famille de substitution confirme d'ailleurs que l'absence des parents est considérée comme le principal traumatisme vécu par les orphelins. Plus que l'expérience de la persécution et de la violence de la guerre, c'est bien la perte des parents qui définit ces individus comme victimes et constitue le moment charnière dans la chronologie intime de leur guerre ${ }^{27}$. Jenoe J. «n'a pu s'installer à Satu Mare parce tout lui rappelait la perte de ses parents et les jours heureux de son enfance ${ }^{28} »$. Serena G. dit que « ses parents et ses frères et sœurs lui manquent terriblement ${ }^{29}$ ». Josef R. écrit : « Nous sommes incapables de vivre ici en paix et heureux. Chaque recoin de notre maison et de ses alentours nous rappelle les êtres chers que nous avons perdus ${ }^{30}$. » Le deuil, part essentielle de ce «bouleversement général de l'univers enfantin ${ }^{31} \gg$ que représente la guerre, est donc essentiellement mis en avant pour justifier la décision de partir.

Dans quelques dossiers, un lien entre l'expérience de la persécution et la personnalité de l'orphelin est plus directement établi. C'est le cas de Pola E. : « À cause de son expérience des camps de concentration et la perte terrible de ses proches, elle n'a pas encore pu déterminer ce qu'elle voulait faire dans le futur ${ }^{32}$. » À propos d'Eva K. : «La terrible existence que cette enfant a menée pendant la guerre a en quelque sorte aiguisé son caractère ${ }^{33}$. » Mais, dans la plupart des cas, ce lien ne semble pas être fait. Plusieurs travailleuses sociales et humanitaires notent certes la « réserve », l'«attitude fataliste », la « timidité » ou la « lenteur» de certains jeunes, comme pour Abraham B., garçon «timide et peu énergique ${ }^{34} »$. Ce vocabulaire renvoie au concept $d^{\prime}$ ' apathie » ou de passivité des personnes déplacées (DP apathy) qui est fréquemment mis en avant par l'administration humanitaire et où le réfugié est pensé comme un «bénéficiaire » (recipient) « intrinsèquement passif et apathique ${ }^{35}$ » ayant perdu toute notion d'autonomie après des années de ségrégation et de promiscuité ${ }^{36}$. D'autres dossiers s'attardent plus sur la maturité précoce des orphelins. Bela L. est décrit comme «un garçon calme et mature ${ }^{37}$ » et Helene H., âgée de 13 ans au moment de son entretien, comme une «jeune fille mature ${ }^{38} »$. Cette maturité est parfois présentée négativement comme chez Tibor I., présenté comme un garçon « agressif » et « trop

\footnotetext{
${ }^{24}$ CJA, dossier individuel de Meyer W., 20 novembre 1947, boîte 41.

${ }^{25}$ CJA, dossier individuel de Martin H., 19 février 1948, boîte 35.

${ }^{26}$ CJA, dossier individuel de Maria K., 4 octobre 1947, boîte 35.

${ }^{27}$ Sur cette chronologie intime de l'enfant, voir Nicholas Stargardt, Witnesses of War: Children's Lives Under the Nazis, New York, Alfred A. Knopf, 2006, p. 12.

${ }^{28}$ CJA, dossier individuel de Jenoe J., 15 mars 1948, boîte 35.

${ }^{29}$ CJA, dossier individuel de Serena G., s. d., boîte 34.

${ }^{30}$ CJA, dossier individuel de Joseph R., 23 février 1948, boîte 38.

31 Manon Pignot, «Expériences enfantines du deuil pendant et après la Grande Guerre », Histoire@Politique : politique, culture, société, 3, 2007, p. 1-12, p. 2.

${ }^{32}$ CJA, dossier individuel de Pola E., 23 février 1948, boîte 32.

${ }^{33}$ CJA, dossier individuel de Eva K., 19 décembre 1947, boîte 36.

${ }^{34}$ CJA, dossier individuel d'Abraham B., 9 janvier 1948, boîte 32.

35 Silvia Salvatici, "Help the People to Help Themselves": UNRRA Relief Workers and European Displaced Persons », Journal of Refugee Studies, 25 (3), 2012, p. 428-451, p. 430.

${ }^{36}$ Sur cette notion qui n'est pas née avec la guerre et qui est encore utilisée par de nombreux travailleurs humanitaires, voir Daniel G. Cohen, In War's Wake: European Refugees in the Postwar Order, New York, Londres, Oxford University Press, 2011, p. 155 ; Peter Gatrell, The Making of the Modern Refugee, Oxford, Oxford University Press, 2013, p. 104-105 ; William I. Hitchcock, The Bitter Road to Freedom : A New History of the Liberation of Europe, Londres, Faber \& Faber, 2009, p. 251-252.

${ }^{37}$ CJA, dossier individuel de Bela L., 30 septembre 1947, boîte 37.

${ }^{38}$ CJA, dossier individuel d'Helene H., 12 décembre 1947, boîte 35.
} 
confiant en lui », « ayant un besoin urgent d'être indépendant et de côtoyer des adultes ${ }^{39}$ ». Ces constats, qu'ils concernent l'apathie, la maturité précoce ou l'indépendance farouche des jeunes survivants, ne semblent que rarement être reliés à la violence et au traumatisme. La guerre est mise entre parenthèses et apparaît comme un passé déjà lointain. Pour des individus comme Edvard F. qui, bien que déporté à Dachau, «a récupéré doucement et est redevenu celui qu'il était avant ${ }^{40} »$, le traumatisme est déjà oublié. On est donc face à une administration qui n'est pas aveugle au processus de déportation et d'extermination puisqu'elle l'atteste et parfois le met en avant. Mais cette reconnaissance est singulière puisque la persécution et la souffrance qu'elle suscite sont rapidement mises entre parenthèses au profit de ce qui constitue un des objectifs sous-jacents du dossier: planifier le retour de l'orphelin à une vie dite «normale».

\section{Rompre avec le passé et rétablir les normes}

Cette ambition normalisatrice est omniprésente dans les dossiers. Elle est une des caractéristiques constituantes du dossier individuel qui doit permettre aux relais du pouvoir institutionnel et bureaucratique de «ramener leurs clients vers les normes sociales et politiques dominantes ${ }^{41} \gg$. Elle est d'autant plus marquée à la sortie de la guerre dans une société obsédée par l'idée de « retour à la normale». Ce que le psychologue israélien Dan Bar-On appellera plus tard «normalisation»- processus où les phases de deuil, de restauration de la confiance en soi et aux autres sont «reportées à des temps plus favorables ${ }^{42} \gg-$ apparaît régulièrement dans les dossiers des orphelins. Ses manifestations évoluent peu entre le début du projet en 1947 et sa fin en 1952. L'émigration y est toujours présentée comme une rupture avec le passé nécessaire afin de laisser définitivement derrière soi les horreurs de la guerre. Le projet de départ est d'ailleurs souvent justifié par la volonté de quitter à tout prix un continent désormais synonyme de persécution et de mort des proches. Lia R. et son frère veulent ainsi « émigrer ensemble et vivre dans la joie, ce qui leur permettra peut-être d'oublier toutes leurs souffrances ${ }^{43} »$. Pour expliquer sa décision de partir au Canada, Tibor K. écrit : "J'espère être libéré de mes souvenirs déprimants du passé ${ }^{44}$. » Les dossiers de ces jeunes empruntent au champ lexical classique des migrants - « opportunité », « rêve », « chance », «futur »-, mais font également souvent référence aux notions d'oubli, de libération, de recommencement et de rupture. Des formules comme «nouvelle vie» ou «nouveau départ » sont parmi les plus utilisées. Le dossier de Jeno S. indique ainsi qu'il «a perdu tous ceux qui lui sont chers et [qu'] il souhaite commencer une nouvelle vie ${ }^{45}$ » et Judith P. dit «se réjouir fortement à l'idée de recommencer sa vie ${ }^{46}{ }$. Le départ au Canada est perçu comme une table rase nécessaire pour l'individu, première étape de sa «normalisation ». Cette volonté de rupture, si elle est parfois présentée comme émanant des jeunes eux-mêmes, est partagée par les travailleuses sociales et humanitaires. Par exemple, dans le dossier de Pola E., s'inquiétant des difficultés de la jeune fille à trouver un projet professionnel lui convenant, la travailleuse humanitaire note : «Nous pensons qu'un nouveau

\footnotetext{
${ }^{39}$ CJA, dossier individuel de Tibor I., 4 août 1948, boîte 35.

${ }^{40}$ CJA, dossier individuel d'Edvard F., 3 avril 1948, boîte 33.

${ }^{41}$ F. Iacovetta et W. Mitchinson, «Introduction », art. cité, p. 6 ; sur cette question, voir aussi le dossier « Le rôle des administrations centrales dans la fabrication des normes », Droit et société, 79 (3), 2011.

${ }^{42}$ Dan Bar-On, « Attempting to Overcome the Intergenerational Transmission of Trauma : Dialogue Between Descendants of Victims and of Perpetrators », in Roberta J. Apfel et Bennett Simon (dir.), Minefields in Their Hearts : The Mental Health of Children in War and Communal Violence, New Haven, Yale University Press, 1996, p. 168.

${ }^{43}$ CJA, dossier individuel de Lia R., 23 février 1948, boîte 38.

${ }^{44}$ CJA, dossier individuel de Tibor K., 22 mars 1948, boîte 36.

${ }^{45}$ CJA, dossier individuel de Jeno S., 17 août 1948, boîte 39 .

${ }^{46}$ CJA, dossier individuel de Judith P., 14 janvier 1948, boîte 38.
} 
départ au Canada lui permettra d'y voir plus clair dans son orientation ${ }^{47}$. » Cette idée de « renouveau » est omniprésente à la sortie de la guerre, surtout à propos de la jeunesse. On la retrouve chez la plupart des décideurs politiques et responsables humanitaires européens. Elle est aussi récurrente dans les milieux pédagogiques juifs. Les dirigeants d'associations comme l'CEuvre de secours aux enfants (OSE) promeuvent ainsi des discours qui font des enfants survivants une "génération libre de toute attache [suppression note de bas de page] », des « buvards » et des « pages blanches » sur lesquels le passé n'a pas laissé de marque durable ${ }^{48}$. Cette idée de renouveau symbolise les espoirs de la reconstruction qui sont placés dans la jeunesse. Elle est aussi une manifestation plus insidieuse de l'injonction faite aux survivants à se taire, à oublier et à avancer ${ }^{49}$.

Le "retour à la normale » tel qu'il se donne à voir dans les dossiers est donc paradoxal puisqu'il comprend à la fois une rupture et un retour, un rejet presque total du passé et un rétablissement des normes de l'existence d'avant-guerre des orphelins. Ainsi, le bon développement des jeunes rescapés est toujours pensé sous la supervision de parents, ce qui sous-entend une restauration de l'environnement familial perdu pendant la guerre. Dans ses négociations avec les autorités canadiennes, le CJC s'est engagé à placer tous les jeunes rescapés en famille d'accueil. L'institution, jugée dépassée, est catégoriquement rejetée. Les travailleuses sociales et humanitaires recommandent toujours le placement en famille, même pour les jeunes approchant ou ayant déjà dépassé la majorité. Cette constante fait écho à la conviction largement partagée dans les rangs de l'AJDC ou de l'IRO, que la famille, consacrée par la Déclaration universelle des droits de l'homme de 1948, est indispensable à la reconstruction d'enfants dont l'individualité a été niée par la guerre. Pour le CJC et ses collaborateurs, les orphelins ont été trop longtemps privés de parents et doivent retourner au plus vite dans un environnement familial, comme Margit G. qui « est impatiente de vivre dans un environnement normal et d'avoir une vie de famille ${ }^{50}$ », ou Rosel K. qui « a besoin de tendresse et d'une vie de famille heureuse pour se réaliser pleinement ${ }^{51}{ }$. Les placements doivent donc permettre aux jeunes de recouvrer un sentiment de sécurité et de faciliter leur « retour à la normale », contribuant ainsi au rétablissement rapide des rôles traditionnels d'âge et de genre.

\section{Restaurer l'enfance et la famille}

Les travailleuses sociales et humanitaires se montrent toujours confiantes quant à l'impact positif du placement sur le développement futur de l'orphelin. Estera H. « aura besoin d'aide lors des premiers mois au Canada parce qu'elle est un peu timide », mais «progresse à force d'encouragements ${ }^{52} »$. Marcel F. «a besoin d'une main ferme pour le guider et l'empêcher de laisser libre cours à son imagination ${ }^{53}$ ». Après avoir souligné le caractère renfermé d'Eva K., celle qui remplit son dossier ajoute que, « dans une atmosphère familiale heureuse, cette dureté disparaîtra sans aucun doute ${ }^{54}$ ». Quant à Janka S., « elle a besoin de beaucoup d'affection et d'attention, il serait donc avisé de la placer dans une famille sans enfant. La

\footnotetext{
${ }^{47}$ CJA, dossier individuel de Pola E., 23 février 1948, boîte 32.

${ }^{48}$ Katy Hazan, Les Orphelins de la Shoah : les maisons de l'espoir (1944-1960), Paris, Les Belles Lettres, 2000, p. 234.

49 Sur cette injonction à commencer une nouvelle vie et à tourner le dos au passé, voir Amos Friedland, «Imre Kertész, Hegel, and the Philosophy of Reconciliation », in Louise Olga Vasvári et Steven Tötösy de Zepetnek (dir.), Imre Kertész and Holocaust Literature, West Lafayette, Purdue University Press, 2005, p. 5164.

${ }^{50}$ CJA, dossier individuel de Margit G., 30 septembre 1947, boîte 34.

${ }^{51}$ CJA, dossier individuel de Rosel K., 19 décembre 1947, boîte 36.

${ }^{52}$ CJA, dossier individuel d'Estera H., 16 juin 1948, boîte 35.

${ }^{53}$ CJA, dossier individuel de Marcel F., 25 septembre 1947, boîte 33.

${ }^{54}$ CJA, dossier individuel d'Eva K., 19 décembre 1947, boîte 36.
} 
sympathie et la confiance seront nécessaires pour qu'elle se débarrasse de sa méfiance et de son attitude fataliste ${ }^{55} \gg$. Le placement en famille apparait donc comme la solution aux difficultés rencontrées par certains orphelins. Dans les dossiers, le processus de normalisation, tel que le définit Bar-On, se manifeste de manière singulière. Le possible traumatisme de l'orphelin n'est pas complètement nié mais n'est jamais présenté comme insurmontable. Un départ au Canada et un bon accompagnement sur place semblent toujours suffisants pour aider le jeune à s'intégrer et à devenir un citoyen utile.

Une des manifestations les plus radicales de cette volonté de normalisation se lit dans la place accordée aux parents d'accueil. Si cela n'est jamais explicitement affirmé dans les dossiers ou dans la correspondance des responsables du CJC et de leurs collaborateurs, l'association juive canadienne a bien l'ambition de trouver des parents de substitution pour les orphelins. Les travailleuses sociales et humanitaires emploient d'ailleurs régulièrement les termes de «nouveaux parents » ou de «nouvelle famille ». La question de l'adoption revient souvent et est même décrite dans les directives du projet comme «l'objectif principal ${ }^{56}$ ». Elle est régulièrement suggérée par des annotations en fin de dossier (« recommandé pour adoption », «adoption si possible » ou «placement en famille pour adoption finale »). Cet espoir semble d'ailleurs être partagé par beaucoup de jeunes, plusieurs orphelins en faisant même la demande expresse. Sandor G., qui a pourtant 17 ans au moment de sa demande de visa, « veut être adopté par une famille qui comprendra pleinement comment s'occuper [de lui] après tant d'années de souffrance et de persécution ${ }^{57}$ ». Quant à Maria K., dont le dossier a été rempli moins de deux mois avant son dix-huitième anniversaire, elle veut être placée «pour adoption dans une famille qui saura parfaitement comment prendre en main la jeune fille après ses années de souffrance ${ }^{58} \gg$. Le rôle des nouveaux parents ne se limite donc pas à accueillir et à accompagner les jeunes dans leur intégration socio-économique au Canada. La famille d'accueil doit non seulement comprendre, mais surtout aider le jeune à oublier son passé. Ce sont également les termes qu'emploie Josef G. qui «espère trouver un nouveau foyer et oublier tout ce qu'il a vu en Europe pendant la guerre ${ }^{59} \gg$. Le vocabulaire utilisé ( « souhaite », « espère » ou « voudrait ») laisse penser que les orphelins font clairement part de leur envie de vivre à nouveau dans un environnement familial et les recommandations des adultes sont toujours présentées comme étant en accord avec la volonté de l'enfant. La travailleuse humanitaire qui remplit le dossier d'Emeric G. écrit d'ailleurs : «J'approuve les projets avancés par l'enfant ${ }^{60}$. $»$ Cette apparente unanimité dans les dossiers quant au placement en famille d'accueil passe sous silence l'impossibilité de remplacer des parents disparus dans des circonstances tragiques, la perte de confiance en l'adulte née de l'impuissance et de l'incapacité de ce dernier à protéger et à survivre ${ }^{61}$. Elle amène à s'interroger sur la place de l'orphelin dans la mise en récit par un tiers dont il est l'objet.

\footnotetext{
${ }^{55}$ CJA, dossier individuel de Janka S., 26 septembre 1947, boîte 39.

56 CJA, «General Features of Planning Project for Immigration : Reception and Placement of 1000 Orphaned Children (PC 1647) », avril 1947, boîte 3 1948/38.

${ }^{57}$ CJA, dossier individuel de Sandor G., 30 septembre 1947, boîte 34.

${ }^{58}$ CJA, dossier individuel de Maria K., 4 octobre 1947, boîte 35.

${ }^{59}$ CJA, dossier individuel de Josef G., 19 novembre 1947, boîte 34.

${ }^{60}$ CJA, dossier individuel d'Emeric G., 19 août 1948, boîte 34.

${ }^{61}$ Sur la perte de confiance dans les adultes née de leur impuissance dans les camps, voir Izio Rosenman, «Life during the Camps and After : Displacement and Rehabilitation of the Young Survivors », in Mandred Gerstenfeld et Françoise Ouzan (dir.), Postwar Jewish Displacement and Rebirth : 1945-1967, Leyde, Brill, 2014, p. 101.
} 


\section{Les « éclats de réel » des dossiers}

La remarque de la travailleuse sociale qui, en conclusion du dossier d'Emeric G., dit « [approuver] les projets avancés par l'enfant ${ }^{62}$ », tout comme l'emploi de la première personne dans certains formulaires, met en évidence une question qui revient inlassablement face à cette archive : qui parle vraiment dans le dossier ? La réponse est de prime abord simple. Les mots des dossiers sont avant tout ceux des travailleuses sociales et humanitaires qui écoutent le jeune survivant, orientent l'entretien et remplissent pour lui sa demande en fonction des exigences définies par une administration dont elles se font les intermédiaires. Même les formulaires où le «je » est employé ne doivent pas faire oublier les possibles erreurs et incompréhensions mais aussi et surtout la marge de manœuvre dont disposent celles qui constituent les dossiers. Comme dans la plupart des processus de demande d'asile et de migration, leur rôle est non seulement de retranscrire et de traduire mais aussi d'interpréter, de reformuler, de formater et de réorganiser chronologiquement le récit de chaque jeune rescapé afin de le rendre intelligible et compatible avec les attentes explicites et implicites de l'administration ${ }^{63}$. Pourtant, peut-on saisir la parole de l'enfant au-delà de l'intervention et de l'écriture des adultes ? Le dossier peut-il devenir un moyen d' " accès unique aux modes de pensée et aux émotions d'enfants qui sortaient à peine de la guerre et de l'Holocauste ${ }^{64} » ? \mathrm{Si}$ cette source ne peut en aucun cas être considérée comme une «source enfantine ${ }^{65}$ », elle se rapproche un peu plus des récits recueillis pendant ou immédiatement après la guerre, en Pologne notamment ${ }^{66}$, bien qu'elle n'en partage pas le désir de «faire preuve » et de «constituer un récit historique collectif» ${ }^{67}$. "J'espère être libéré de mes souvenirs déprimants du passé ${ }^{68} \gg$ est ainsi une retranscription de la travailleuse sociale qui se fait à la fois traductrice de Tibor K. qui ne parle que le hongrois et relais face à l'administration. Il en est de même pour Marko R. qui se remémore « la faim, le froid et la terreur ${ }^{69}$ ». Le dossier n'est donc pas à proprement parler un témoignage puisqu'il n'en a pas la finalité mémorielle

${ }^{62}$ CJA, dossier individuel d'Emeric G., 19 août 1948, boîte 34.

${ }^{63}$ Pour un éclairage contemporain sur cette question, outre les travaux de Didier Fassin, voir par exemple Chiara Galli, «A Rite of Reverse Passage : The Construction of Youth Migration in the US Asylum Process », Ethic and Racial Studies, 2017 (prépublication en ligne).

64 Joanna B. Michlic, «What Does a Child Remember? Recollections of the War and the Early Postwar Period among Child Survivors from Poland », in Joanna B. Michlic (dir.), Jewish Families in Europe, 1939Present : History, Representation, and Memory, Waltham, Brandeis University Press, 2017, p. 153-172, p. 153.

65 Manon Pignot, «Génération Grande Guerre : expériences enfantines du premier conflit mondial », Le Télémaque, 47 (2), 2012, p. 77 ; Célia Keren, «Autobiographies of Spanish Refugee Children at the Quaker Home in La Rouvière (France, 1940) : Humanitarian Communication and Children's Writings », Les Cahiers de FRAMESPA, 5, 2010.

66 Joanna Michlic, «Rebuilding Shattered Lives: Some Vignettes of Jewish Children's Lives in Early Postwar Poland», in Dalia Ofer, Françoise S. Ouzan et Judy Tydor Baumel-Schwartz (dir.), Holocaust Survivors : Resettlement, Memories, Identities, New York, Oxford, Berghahn Books, 2011, p. 46-87 ; Joanna Michlic, "The War Began for Me after the War": Jewish Children in Poland, 1945-1949», in Jonathan Friedman (dir.), The Routledge History of the Holocaust, Londres, Routledge, 2011, p. 482-497 ; Boaz Cohen, «The Children's Voice : Postwar Collection of Testimonies from Child Survivors of the Holocaust », Holocaust and Genocide Studies, 21 (1), 2007, p. 73-95; Sharon Kangisser Cohen, «A Child's View: Children's Depositions of the Central Jewish Historical Commission (Poland) », in Sharon Kangisser Cohen, Eva Fogelman et Dalia Ofer (dir.), Children in the Holocaust and its Aftermath: Historical and Psychological Studies of the Kestenberg Archive, New York, Berghahn Books, 2017, p. 43-61.

${ }^{67}$ Audrey Kichelewski et Judith Lindenberg, "'Les enfants accusent": témoignages d'enfants survivants dans le monde polonais et yiddish », in Ivan Jablonka (dir.), L'Enfant-Shoah, Paris, PUF, 2014, p. 33-50, p. 42.

${ }^{68}$ CJA, dossier individuel de Tibor K., 22 mars 1948, boîte 36.

${ }^{69}$ CJA, dossier individuel de Marko R., 23 février 1948, boîte 38. 
ou judiciaire et participe seulement de la « définition de soi administrative ${ }^{70} »$ que nécessite la constitution de la demande de visa.

Pourtant, il représente sûrement pour beaucoup d'orphelins une première opportunité de se remémorer et un espace rare où ils ont pu parler et être écoutés. Ces mots renseignent donc aussi sur leurs émotions et leurs représentations. La narration de leur guerre et de la séparation d'avec leurs parents, la justification du départ et les projets d'avenir laissent entrevoir les souffrances, le deuil et le traumatisme des jeunes rescapés ainsi que les attentes et les espoirs que suscite chez eux la possibilité d'un départ au Canada. Les quelques récits plus détaillés confirment d'ailleurs aussi la volonté de certains jeunes de raconter leur expérience de la persécution. De la même manière, la connaissance relativement précise du génocide qui transparaît dans les dossiers est certes le fait de celles qui remplissent les formulaires et qui orientent l'entretien par leurs questions, mais elle reflète aussi une « conscience historique détenue par les enfants ${ }^{71}$ » qui participent à la mise en récit de leur expérience et à la reconstruction de leur trame biographique. Tout en ayant à l'esprit les règles qui encadrent cette « obligation de se raconter», les dossiers n'en restent donc pas moins des récits précoces, et pour cela précieux, qui laissent entrevoir un «monde caché ${ }^{72}$ » presque toujours hors de portée de l'historien. Ces « éclats de réel ${ }^{73}$ » nous éclairent sur les destins exceptionnels des orphelins, sur leurs modes de pensée et sur leurs émotions. Ils révèlent aussi la capacité de ces jeunes à résister ou, du moins, à influencer la nature de leur relation avec l'administration et ses intermédiaires ${ }^{74}$. L'analyse d'un tel corpus permet dès lors de contribuer aux efforts récents $d$ ' "examiner les pratiques des migrants eux-mêmes ${ }^{75}$ », d'écrire une histoire des réfugiés par le bas et surtout de sortir les enfants en guerre du " rôle singulièrement passif ${ }^{76} \gg$ dans lequel ils ont été longtemps enfermés. Elle participe surtout d'une ambition plus globale qui résonne singulièrement dans l'inquiétude de la fin de l'« ère des témoins ", celle d'écrire une histoire des survivants de la Shoah et de leurs expériences par les archives, une histoire sociale qui s'autoriserait enfin à appréhender ces trajectoires exceptionnelles au prisme des méthodologies classiques de la discipline.

Antoine Burgard, University of Manchester, Humanitarian and Conflict Response Institute, M13 9PL, Manchester, Royaume-Uni.

Antoine Burgard est titulaire d'un doctorat en histoire de l'Université Lyon-II et de l'Université du Québec à Montréal. Il est actuellement chercheur postdoctoral à l'Humanitarian and Conflict Response Institute (University of Manchester) et boursier de la Fondation pour la Mémoire de la Shoah. Il conduit en parallèle un projet de collecte de témoignages de survivants de la Shoah au Canada, en France et au Royaume-Uni avec la Fondation Claude-Levy enfant juif caché (Strasbourg). (burgard.antoine@ gmail.com)

${ }^{70}$ Xavier Crettiez et Pierre Piazza (dir.), Du papier à la biométrie : identifier les individus, Paris, Presses Science Po, 2006, p. 20.

${ }^{71}$ A. Kichelewski et J. Lindenberg, «"Les enfants accusent”... », art. cité, p. 41.

${ }^{72}$ Zeev Mankowitz, Life Between Memory and Hope : The Survivors of the Holocaust in Occupied Germany, Cambridge, Cambridge University Press, 2002, p. 22.

${ }^{73}$ L. Bantigny et J.-C. Vimont, « Observer les observateurs... », art. cité, p. 8.

${ }^{74}$ Sur les stratégies de présentation de soi, voir D. Fassin, «La supplique... », art. cité ; sur la capacité de l'usager (client) à influencer son «expérience du contrôle social», voir Linda Gordon, "Family Violence, Feminism, and Social Control », Feminist Studies, 12 (3), 1986, p. 452-478; pour une discussion à ce sujet, voir les échanges entre Joan W. Scott et Linda Gordon dans Signs, 15 (4), 1990.

75 Paul-André Rosental, «Migrations, souveraineté, droits sociaux : protéger et expulser les étrangers en Europe du XIX ${ }^{\mathrm{e}}$ siècle à nos jours », Annales : histoire, sciences sociales, 66 (2), 2011, p. 335-373, p. 352.

${ }^{76}$ Nicholas Stargardt, «Jeux de guerre : les enfants sous le régime nazi », Vingtième Siècle : revue d'histoire, 89, janvier-mars 2006, p. 61-76, p. 69. 